\title{
Self-Assembled Stripe Patterns of CdS Nanorods
}

\author{
Ali Ghezelbash and Brian A. Korgel* \\ Department of Chemical Engineering, Texas Materials Institute, \\ Center for Nano- and Molecular Science and Technology, \\ The University of Texas at Austin, Austin, TX 78712-1062 \\ *Corresponding author: korgel@che.utexas.edu; T: (512) 471-5633; F: (512) 471-7060
}

CdS Nanorod Synthesis. ${ }^{1}$ On a Schlenk line using standard airless procedures, $0.23 \mathrm{~g}$ (1.8 mmol) $\mathrm{CdO}$ (Aldrich) was added to $0.83 \mathrm{~g}(3.0 \mathrm{mmol}) \mathrm{N}$-tetradecylphosphonic acid (TDPA, Alfa Aesar) and 7g trioctylphosphine oxide (TOPO, Strem), and heated for $1 \mathrm{~h}$ at $80{ }^{\circ} \mathrm{C}$ under nitrogen. Separately, 0.18g $(4.7 \mathrm{mmol})$ elemental sulfur (Aldrich) was dissolved in $20 \mathrm{~mL}$ of trioctylphosphine (TOP, Strem). The $\mathrm{Cd}$ mixture was heated to $340{ }^{\circ} \mathrm{C}$ to fully complex $\mathrm{Cd}$ with TDPA, as indicated by a color change from dark red to clear and colorless, then cooled to 300 ${ }^{\circ} \mathrm{C}$. $6 \mathrm{~mL}$ of S-TOP solution was injected into the reaction flask under vigorous stirring. After stirring for $1.5 \mathrm{~h}$ at $300{ }^{\circ} \mathrm{C}, 14 \mathrm{~mL}$ of S-TOP solution is injected by syringe pump at a very slow injection rate of $10 \mathrm{~mL} / \mathrm{h}$. Shorter nanorods were obtained by dropping the temperature to 210 ${ }^{\circ} \mathrm{C}$ after the initial injection of $6 \mathrm{~mL}$ of $\mathrm{S}-\mathrm{TOP}$ and stirring for $1.5 \mathrm{~h}$ before raising the temperature back to $300{ }^{\circ} \mathrm{C}$ and injecting an additional $14 \mathrm{~mL}$ of S-TOP by syringe pump. Nanorods were purified by precipitation in excess acetone, followed by centrifugation at 8000 rpm for 4 min. The supernatant was discarded.

Nanorod deposition and TEM imaging. Nanorods were redispersed in chloroform at concentrations ranging from 0.7 to $2.8 \mathrm{mg} / \mathrm{mL}$ and drop cast onto 200 mesh carbon-coated copper TEM grids (Ladd Research). In some depositions, excess solvent was removed by 
wicking with a kim-wipe. Wicking generally helped ensure that the nanorods did not deposit in thick layers and stripes formed regardless of whether this wicking procedure was used. TEM images were acquired using a Philips 208 TEM with $80 \mathrm{kV}$ accelerating voltage with an AMT Advantage HR model CCD Camera.

Estimation of the Hamaker constant. The Hamaker constant for two CdS nanorods interacting across chloroform was estimated from the Hamaker constants for chloroform and CdS across vacuum. For chloroform: ${ }^{2,3}$

$$
A_{22}=\frac{3}{4} k T\left(\frac{\varepsilon_{2}-1}{\varepsilon_{2}+1}\right)^{2}+\frac{3 h v_{e}}{16 \sqrt{2}} \frac{\left(n_{2}{ }^{2}-1\right)^{2}}{\left(n_{2}{ }^{2}+1\right)^{3 / 2}}=0.364 \mathrm{eV}
$$

where the subscript "2" denotes chloroform, $k$ is Boltzmann's constant, $T$ is temperature, $\varepsilon_{2}$ is the dielectric constant (4.8), $n_{2}$ is the refractive index (1.443), $h$ is Planck's constant and $v_{e}$ is the maximum electronic ultraviolet absorption frequency. For CdS, $A_{11}=0.686 \mathrm{eV}{ }^{4}$ The Hamaker constant for CdS nanorods interacting across chloroform is

$$
A_{121} \approx\left(\sqrt{A_{11}}-\sqrt{A_{22}}\right)^{2}=0.0506 \mathrm{eV}
$$

\section{References}

(1) Shieh, F.; Saunders, A. E.; Korgel, B. A. J. Phys. Chem. B 2005, 109, 8538-8542.

(2) Israelachvili, J. N. Intermolecular and Surface Forces, $2^{\text {nd }}$ ed.; Academic Press: London, 1991.

(3) Sigman, M. B.; Saunders, A. E.; Korgel, B. A. Langmuir 2004, 20, 978-983.

(4) Bergstrom, L., Adv. Coll. Int. Sci. 1997, 70, 125-169. 